\title{
Dorsal rhizotomy for children with spastic diplegia of cerebral palsy origin: usefulness of intraoperative monitoring
}

\author{
George Georgoulis, MD, ${ }^{1-3}$ Andrei Brînzeu, MD, MSc, PhD, ${ }^{1,4}$ and Marc Sindou, MD, DSc ${ }^{1,5}$ \\ ${ }^{1}$ University of Lyon, France; ${ }^{2}$ Neurosurgery Department, "Agia Sofia" Children's Hospital of Athens; ${ }^{3}$ Medical School, University \\ of Athens, Greece; "University of Medicine and Pharmacy "Victor Babeș" Timișoara, Romania; and ${ }^{5} \mathrm{Clinique} \mathrm{Bretéché,} \mathrm{Groupe}$ \\ ELSAN, Nantes, France
}

OBJECTIVE The utility of intraoperative neuromonitoring (ION), namely the study of muscle responses to radicular stimulation, remains controversial. The authors performed a prospective study combining ventral root (VR) stimulation for mapping anatomical levels and dorsal root (DR) stimulation as physiological testing of metameric excitability. The purpose was to evaluate to what extent the intraoperative data led to modifications in the initial decisions for surgical sectioning established by the pediatric multidisciplinary team (i.e., preoperative chart), and thus estimate its practical usefulness.

METHODS Thirteen children with spastic diplegia underwent the following surgical protocol. First, a bilateral intradural approach was made to the L2-S2 VRs and DRs at the exit from or entry to their respective dural sheaths, through multilevel interlaminar enlarged openings. Second, stimulation-just above the threshold—of the VR at $2 \mathrm{~Hz}$ to establish topography of radicular myotome distribution, and then of the DR at $50 \mathrm{~Hz}$ as an excitability test of root circuitry, with independent identification of muscle responses by the physiotherapist and by electromyographic recordings. The study aimed to compare the final amounts of root sectioning - per radicular level, established after intraoperative neuromonitoring guidance-with those determined by the multidisciplinary team in the presurgical chart.

RESULTS The use of ION resulted in differences in the final percentage of root sectioning for all root levels. The root levels corresponding to the upper lumbar segments were modestly excitable under DR stimulation, whereas progressively lower root levels displayed higher excitability. The difference between root levels was highly significant, as evaluated by electromyography $(p=0.00004)$ as well as by the physiotherapist $(p=0.00001)$. Modifications were decided in 11 of the 13 patients $(84 \%)$, and the mean absolute difference in the percentage of sectioning quantity per radicular level was $8.4 \%$ for $L-2(p=0.004), 6.4 \%$ for $L-3(p=0.0004), 19.6 \%$ for $L-4(p=0.00003), 16.5 \%$ for $L-5(p=0.00006)$, and $3.2 \%$ for S-1 roots $(p=0.016)$. Decreases were most frequently decided for roots $L-2$ and $L-3$, whereas increases most frequently involved roots L-4 and L-5, with the largest changes in terms of percentage of sectioning.

CONCLUSIONS The use of ION during dorsal rhizotomy led to modifications regarding which DRs to section and to what extent. This was especially true for L-4 and L-5 roots, which are known to be involved in antigravity and pelvic stability functions. In this series, ION contributed significantly to further adjust the patient-tailored dorsal rhizotomy procedure to the clinical presentation and the therapeutic goals of each patient.

https://thejns.org/doi/abs/10.3171/2018.1.PEDS17577

KEYWORDS intraoperative neuromonitoring; dorsal rhizotomy; cerebral palsy; spastic diplegia; spine

$\mathrm{S}$ PASTICITY in diplegic children affected with cerebral palsy (CP) is a pathological condition, the effects of which are cumulative and cause musculoskeletal deterioration, especially during the years of locomotor development, leading to even more severe handicaps. When spasticity becomes refractory to physical therapy and all other treatments such as botulinum toxin injections, neurosurgery may be a recourse to rebalance postures and gait by reducing the excess of muscle tone and preventing deformities.

ABBREVIATIONS CMAP = compound motor action potential; $\mathrm{CP}=$ cerebral palsy; DR = dorsal root; $\mathrm{DRh}=$ dorsal rhizotomy; EMG = electromyographic; IL = interlaminar; ION = intraoperative neuromonitoring; $\mathrm{KIDr}=$ keyhole interlaminar dorsal rhizotomy; $\mathrm{VR}=$ ventral root.

SUBMITTED October 15, 2017. ACCEPTED January 2, 2018.

INCLUDE WHEN CITING Published online April 13, 2018; DOI: 10.3171/2018.1.PEDS17577. 
Since its introduction in 1898 by Foerster ${ }^{14}$ lumbosacral dorsal rhizotomy (DRh) has remained a controversial procedure because it involves lesioning and irreversible effects. The challenge consists in achieving effectiveness and selectivity at the same time. Sectioning too many fibers would compromise useful tone, whereas sectioning too few would result in insufficient effect or potential recurrence. The aim is to target the appropriate radicular levels and thus the proper muscle groups, namely those that harbor harmful spastic components, as well as to interrupt just the appropriate amount of fibers so as not to weaken the muscle groups that sustain useful tone, especially the ones responsible for pelvic stability and antigravity function.

Multidisciplinary teams together with the caregivers should establish a tailored operative plan for each child. Realistic goal setting is the key to a satisfactory outcome, with the postoperative expectations and limits being weighted and explained to the patient and family. All of these factors have to be written down in the form of a preoperative chart.

Various modalities of intraoperative explorations have been developed over decades, based on the following principles: 1) stimulation of the ventral roots (VRs) for verifying the precision of radicular levels; and 2) stimulation of the dorsal roots (DRs) to evaluate the degree of excitability of the metameric circuitries. Intraoperative neuromonitoring (ION) is demanding in terms of duration of surgery and its implications for the anesthesiological and surgical team. What should be the optimal protocol remains controversial. Not only are modalities not uniformly applied among the various teams dealing with this type of surgery, ${ }^{45}$ but also the usefulness of ION itself is a matter of debate. ${ }^{31,43}$

To provide insight in this practical debate, we launched a prospective study in a preliminary series of 13 patients, comparing the decisions written down in the preoperative chart with the data collected from ION. For this work the neurophysiological investigations have been performed in the most complete fashion possible. In this study the perspective was limited to evaluating to what extent the intraoperative information led to modifications in the initial decisions for surgical sectioning, and thus to estimate its practical usefulness.

\section{Methods \\ Patient Selection}

Patients enrolled in this study suffered spastic diplegia of $\mathrm{CP}$ origin, with various degrees of severity. In spite of physical therapy and other nonsurgical interventions, all children experienced deterioration of their locomotor development along with evolving deformities. The main criterion for the decision to perform surgery, and for its timing, was the regression in the Gross Motor Function Measure score. ${ }^{38,41,42}$ For the children able to walk, the goal was to improve ambulation and gait and prevent contractures. For the nonambulatory children, the aim was to ease care, improve comfort, and stop the evolution of deformities. The objectives and limits of the surgery were established within the framework of the institution's multidisciplinary team. The study was approved by the institutional review board of the University of Lyon. Written informed consent was obtained from the families of all 13 patients.

\section{Surgical Planning}

Preoperative Chart

The plan was tailored to each patient's features (Fig. 1) and the objectives were written down in a preoperative chart along with the patient's informed consent. The preoperative chart included the following: 1) description of the harmful components of spasticity; 2) enumeration of the muscle groups harboring excess of tone; 3 ) designation (from L-2 to S-1/S-2 root) of the corresponding DRs expected to carry the "deleterious afferents" and thus to be targeted; and 4) estimation of the percentage of the dorsal rootlets to be cut per targeted DR, according to the excess of tone in the corresponding myotome(s) $(50 \% \pm 25 \%$ per targeted root). ${ }^{42}$ Also, roots corresponding to muscles whose tone needed to be preserved-especially those playing an antigravity role or participating in pelvic stability (principally L-4 for quadriceps and L-5 for gluteus muscles), particularly in ambulatory children, were noted so they could be spared. Sectioning S-2, which would be justified to reduce tone in hamstrings and/or flexors of the toes, was considered with prudence due to involvement of this root in the urinary and genital functions.

\section{Setting for ION and Electromyographic Recordings}

To confirm or modify the surgical plan previously written in the preoperative chart, intraoperative investigations were undertaken, which consisted of the identification and study of the muscle responses after radicular stimulation, both as observed by the physiotherapist and as recorded by electromyography.

Electromyographic Recordings. Patients were placed in the prone position (Fig. 2A). The electromyographic (EMG) exploration included several cardinal muscles in both lower limbs, namely adductors (adductor longus) for L2-L3 roots; quadriceps (rectus femoris) for L3-L4; tibialis anterioris for L-4; extensor hallucis for L-5; hamstrings (biceps femoris) for L5-S2; triceps surae (soleus) for S-1; flexor digitorum brevis for S-2; and external anal sphincter with electrodes placed at the 3rd and 9th hours for S2-S4. The recording electrode made of 2 intramuscular needles was inserted in each selected muscle. The distance between the 2 needles was approximately $2 \pm 1 \mathrm{~cm}$, because a greater distance would risk recruiting neighboring muscle groups and compromise specificity and thus selectivity. The ground electrode was placed in the reference point, subcutaneously-namely at the level of iliac crest on one side.

Compound motor action potentials (CMAPs) were recorded on 16 channels ( 8 successive channels on each limb). The display form had a total vertical deviation (gain) of potential of -500 to $500 \mathrm{mV} /$ division and a sweep spread of $75 \mathrm{msec} /$ division. For root stimulation, a fine flexible bipolar probe was used, and the distance between anode and cathode was $3-5 \mathrm{~mm}$.

The EMG recordings had a real-time mode, i.e., EMG activities were continuously monitored. This was useful 


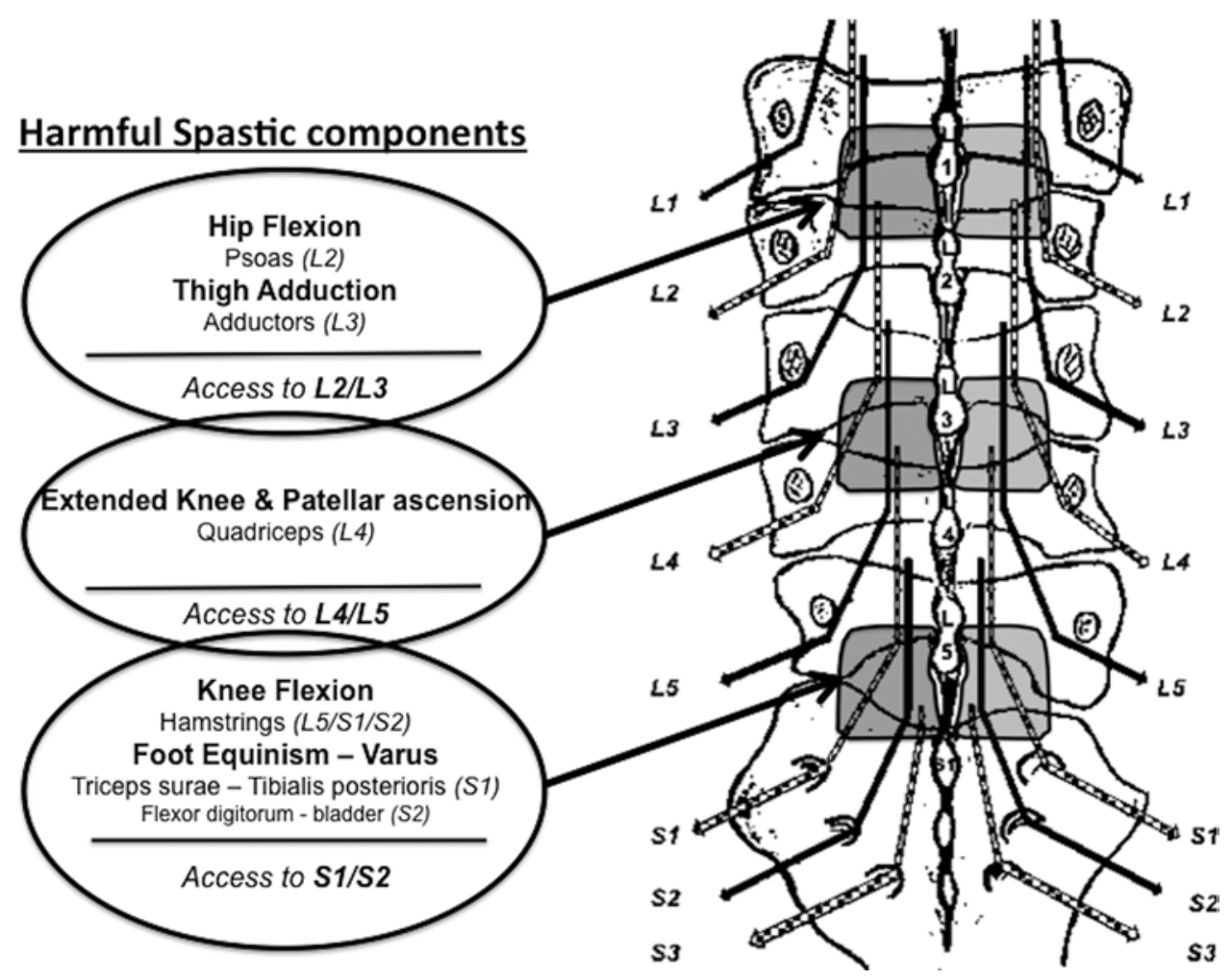

FIG. 1. Schematic drawing of IL vertebral levels where selected roots can be targeted for DRh: at L1-L2 for roots L-2/L-3; at L2-L3 for roots $L-3 / L-4$; at $L 3-L 4$ for roots $L-4 / L-5$; at $L 4-L 5$ for roots $L-5 / S-1$; and at $L 5-S 1$ for roots $S-1 / S-2$. The IL spaces to be opened are determined according to the preoperative plan for root sectioning (i.e., tailored operation). The gray squares represent IL vertebral levels where targeted roots are approached intradurally in this illustration. Roots L-2 and L-3 were targeted for treatment of spastic hip flexion and thigh adduction. Root L-4 was targeted for treatment of extended knee with patellar ascension. Root S-1 was targeted at the L5-S1 IL space for treatment of spastic foot in equinism, varus, and spastic hamstrings.

during radicular dissection. Any disturbance was traced directly so that the roots could be protected from eventual aggressive manipulations.

Physiotherapist Observation. In our protocol the institution's physiotherapist was present throughout the operation. For this purpose a space was organized to enable access to the patient's lower limbs (Fig. 2A). The physiotherapist was asked to evaluate the muscle responses after stimulation of each root, by direct vision and palpation of muscle contractions, independently from the EMG recordings. In muscle groups not accessible to EMG recordings, as for the psoas-iliacus group, the study was based exclusively on the physiotherapist's observation. His or her role was also fundamental for the observation of the eventual responses spreading to the contralateral lower limb or even upper limbs.

\section{Anesthesia}

Surgery was performed with the patient placed prone, under general anesthesia (Fig. 2A). The patients were intubated under rapid-sequence induction, using short-acting neuromuscular agents. No muscle relaxants were administered thereafter, because they would render muscle responses to intraoperative stimulation uninterpretable. For maintenance of anesthesia, the agents used were inhaled sevoflurane (or 50\%-70\% nitrous oxide) and intravenous sufentanil or remifentanil.

\section{Surgical Approach}

The surgical approach was the modality that we have previously published under the name of keyhole interlaminar dorsal rhizotomy (KIDr). ${ }^{41}$ In brief, the method is based on the creation of 2-3 enlarged midline interlaminar (IL) openings (Fig. 2B), whose number and level are decided individually for each patient on the basis of the preoperative chart, as illustrated in the schematic drawing of Fig. 1. The principle of this approach is to respect the spinous processes and interspinous ligaments. At each selected IL space, the flavum ligament is entirely removed. According to our original description, the size of each window (enlarged IL space and midline dural opening) is on the order of $2.5 \mathrm{~cm}$, which generally allows access to 2 adjacent roots (1 upper and 1 lower) by modifying the axis of the microscope (Fig. 2C). Optimal length of the IL opening requires the resection of approximately two-thirds of the upper and three-fourths of the lower laminae. Then dura mater is opened on the midline. According to the keyhole principle, roots are accessed from the contralateral side, passing underneath the preserved interspinous ligament via an oblique intradural trajectory (Fig. 2D-F).

The ventral and dorsal components of each exposed root were identified intradurally at the exit from or entry to their dural sheath (Fig. 2D). The VR and the DR, the latter generally consisting of 4-5 distinct rootlets, were 

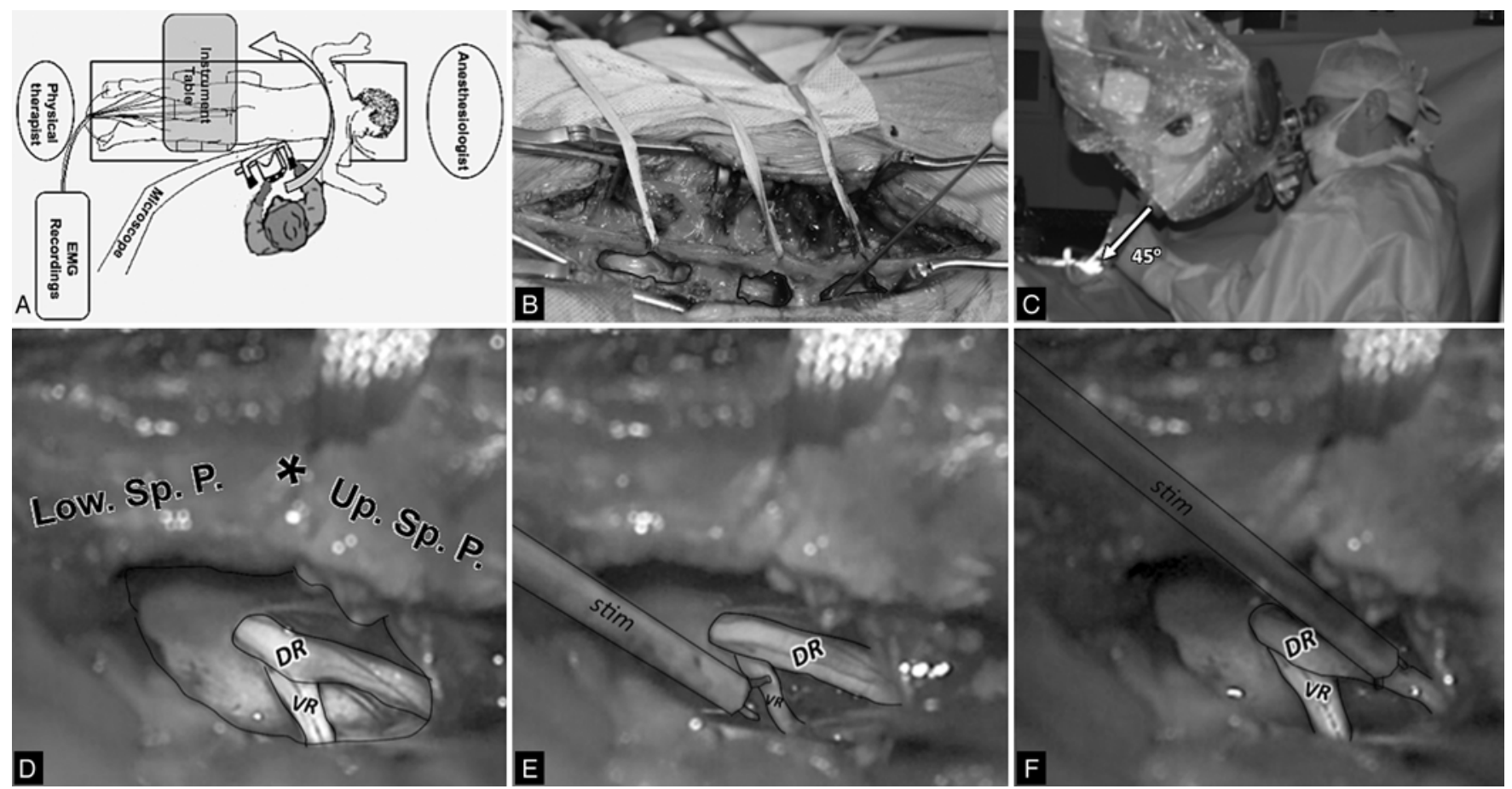

FIG. 2. A: Schematic drawing of the organization of the operating room and surgical team. The curved arrow indicates that the surgeon changes position and side to obtain best access to the targeted root. B: Exposure of L1-S1 laminae, with L1-L2, L3-L4, and L5-S1 IL fenestrations, as selected at surgical planning. At each fenestrated level, the inferior two-thirds of upper lamina and the superior two-thirds of lower lamina are rongeured, and flavum ligamentum is removed to expose dura, so that dura and arachnoid are opened on the midline. Note at each fenestrated level, the preservation of the spinous processes and interspinous ligament (tapes). C: Placement of microscope allows rotation from one side to the other, and targeted roots should be approached from the contralateral side in an oblique $45^{\circ}$ axis. D: Exposure and individualization of the VRs and DRs at the exit from or entry to their dural sheath. At each fenestrated level, the inferior two-thirds of upper lamina and the superior two-thirds of lower lamina and flavum ligamentum are removed to expose dura, so that dura and arachnoid are opened on the midline. At each fenestrated level, the spinous processes and the interspinous ligament (asterisk) are preserved. Enlarged IL space on the order of $2.5 \mathrm{~cm}$. Low. Sp. P. = lower spinous process; Up. Sp. P. = upper spinous process. E and F: Stimulation of the VR (E) and DR (F) performed using a bipolar electrode (stim). Anatomy is characteristic, with the VR being situated anteriorly and the DR posteriorly. The $\mathrm{DR}$ is approximately twice as thick as the VR.

separated at a length that ranged from $5 \mathrm{~mm}$ to no more than $10 \mathrm{~mm}$, to minimize manipulation and thus preserve the roots' electrical conduction properties.

\section{Root Stimulation}

Stimulation of roots was performed with a bipolar probe, with an interpole distance of $4 \pm 1 \mathrm{~mm}$. A few seconds of stimulation, with the probe being kept in close contact with the root, are needed before obtaining a steady state reliable enough to interpret the muscle responses. Three consecutive stimulations provoking similar results are a prerequisite before interpretation of the muscle responses is performed.

Stimulation of VRs (i.e., anatomical mapping) was performed orthodromically to identify their innervation territory (i.e., myotome) and thereby confirm their topographical level (Fig. 2E). The VR stimulation was performed using $2 \mathrm{~Hz}$ of frequency at a low intensity: namely 200 $\mu \mathrm{A}$, which is generally slightly above the motor threshold (currently at $50 \mu \mathrm{A}$ ), so that the risk of producing spread of current to adjacent roots is minimal. The starting point of VR stimulation was at the level of L-2, resulting in hip flexion from the psoas-iliacus muscle and thigh adduction; then stimulation was continued down to S-2/S-3 for checking anal sphincter responses.

Stimulation of DRs (i.e., physiological testing) was the next step, to evaluate their degree of involvement in the tone circuit excitability (Fig. 2F). In this series of patients stimulation of the DR was performed globally (i.e., including all the constituting rootlets as a whole). According to Fasano's description, stimulation was in the form of a 1 -second train of $50-\mathrm{Hz}$ square-wave pulses, each with a $0.1-\mathrm{msec}$ duration, slightly above threshold-generally at $1 \mathrm{~mA}$ of intensity. ${ }^{10-12}$ This intensity, which is approximately 3-5 times above the motor response threshold, was chosen so as not to elicit excessive "parasite" diffusions.

Of note, stimulation of the S-2 DR was included in the protocol, but its purpose was to confirm topography and myotomal distribution, and not to be targeted for surgical sectioning because of its participation in urinary and genital innervation, with just 1 exception (case 3).

Interpretation of DR Stimulation Responses for Surgical Decision Making. The criteria used to distinguish normal from abnormal muscle responses were those originally described by Fasano and colleagues ${ }^{10-12}$ and modi- 

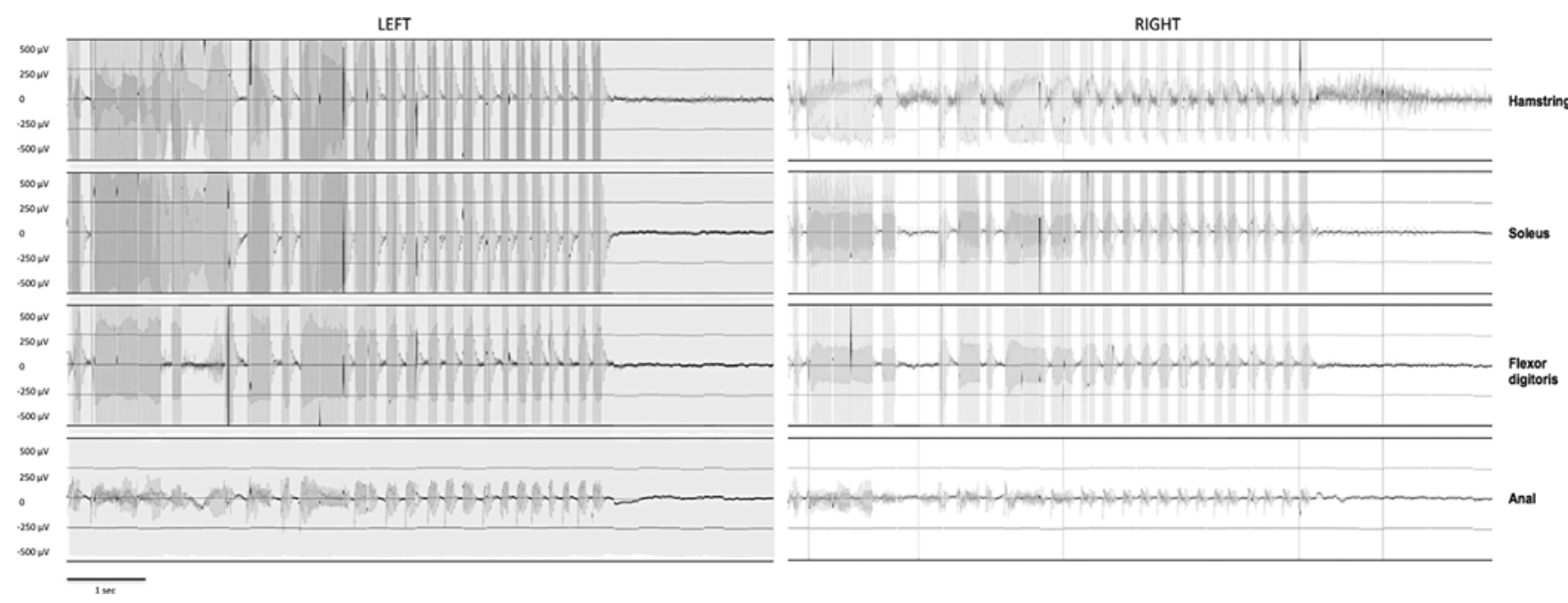

FIG. 3. Intraoperative EMG recordings obtained after stimulation (1 second at $50 \mathrm{~Hz}$ with constant current at $1 \mathrm{~mA}$ ) of the left S-1 dorsal root in a 7 -year-old diplegic child (case 9). The CMAPs were recorded from intramuscular electrodes placed bilaterally on various muscle groups of the lower limbs. The recordings show diffusion of response to the contralateral lower extremity after stimulation. The maximally abnormal grade of 4 was assigned to this EMG response pattern. Note the recorded diffused incremental, multiphasic, and clonic response.

fied by Philips and Park ${ }^{35,36}$ and Mittal. ${ }^{21}$ Responses were estimated to be normal (grade 0) when located in the muscle group(s) corresponding to the segmental level of the stimulated DR (i.e., myotome) and with a single motor response-or with multiple responses but having a decremental amplitude pattern. Responses were considered abnormal when characterized by an incremental amplitude pattern or sustained as follows: in the corresponding myotome (grade 1), in myotomes of adjacent level(s) (grade 2), or in myotomes distant from that of the stimulated DR (grade 3). Grade 4 is assigned when spreading responses are diffusing to muscles in the contralateral lower limb or the upper limb(s) (Fig. 3). ${ }^{16,17,21}$

According to the EMG recordings and the physiotherapist's observations, which were independent from each other, the motor response of each root was assigned a grade of excitability of $0,1,2,3$, or 4 , as shown in Table 1 .

Criteria for Root Sectioning. Responses to stimulation were used to determine which DRs and which amount of rootlets per root were to be cut. The final decision to respect or transect and to what extent (i.e., the operative sectioning plan) was established from the synthesis of the 3 following elements: 1) the preoperative chart; 2) the electrophysiological responses monitored through the 16-channel recordings ( 8 from each side); and 3 ) the muscle contractions as documented by the physiotherapist.

The surgical plan established preoperatively was thus adjusted according to the intraoperative information. Increases in the percentage of DR sectioning were decided when grades were found to be 4 or 3 , and decreases when grades were 2 or 1 .

\section{Data Analysis}

Data were recorded in Excel spreadsheets. Analysis mainly consisted of the comparison of the preoperative planning with the final decisions for sectioning made on the basis of ION information. Modifications in the surgical plan were quantified by calculating the difference between the final percentage of roots that were sectioned and the percentage of the preoperative estimation. Depending on whether the final sectioning percentage was higher, lower, or equal to the initial estimate, the difference was positive, negative, or none.

The study included both the overall modifications in the 130 total targeted roots $(13$ patients $\times 5$ levels $\times 2$ sides) and the modifications with regard to each radicular level. Of note, data from the stimulation of S-2 roots have not been used in the calculations, except for the patient in case 3 . In the patient in case 5 we proceeded to the KIDr technique by performing only 2 IL fenestrations, at levels L1-L2 and L4-L5, so that the L-4 roots were not approached and therefore not stimulated.

The values calculated were as follows: 1) the percentage of patients in whom modification was decided after ION; 2) the percentage of roots in which modification was decided and the individual percentages of roots that underwent an increase or decrease in sectioning; 3) the overall magnitude of the modifications, expressed as the mean absolute value of the difference between the initially decided and the final (after ION) percentage of sectioning for all roots, and also the mean increase in sectioning in the subgroup of roots in which an increase was decided and, similarly, the mean decrease in sectioning; 4) the percentage and magnitude (mean absolute difference) of increases and decreases for each radicular level, from L-2 to $S-1 ; 5)$ the various grades of excitability, according to Fasano et al.'s classification, per radicular level; and 6) the concordances and/or discordances between the findings of the physiotherapist and the EMG recordings, and their important role in the decision about sectioning.

The systematic radicular exploration, performed for practical reasons on each of the 6 exposed VRs (from L-2 to S-2) on both sides, provided additional knowledge about their myotomal distribution, as well as the symmetry be- 


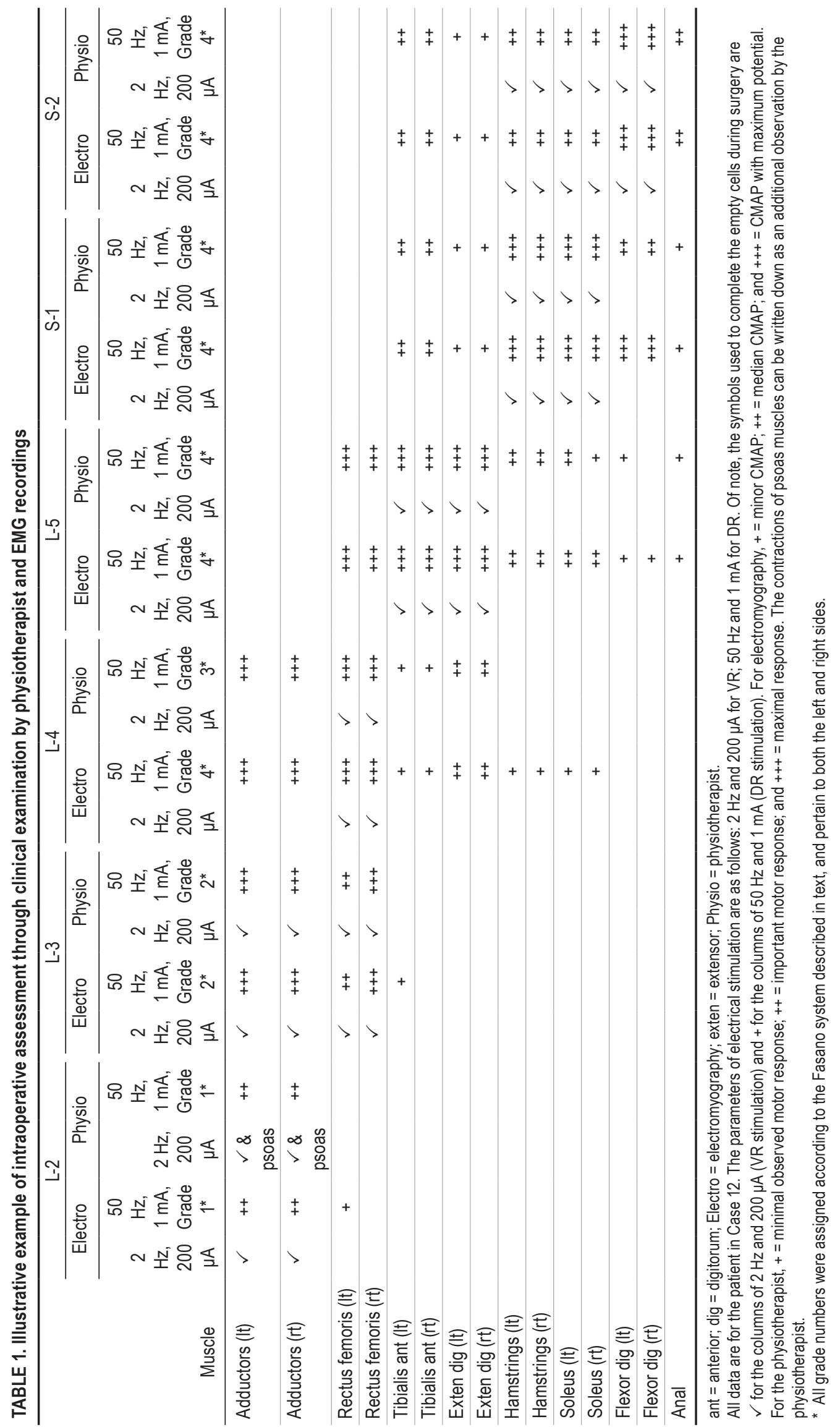


tween the left and right side in myotomal topography. The same exploration was performed using DR stimulation. The data corresponding to the results of this functional anatomical study are the subject of another work that is currently under preparation.

\section{Statistical Analysis}

We checked whether the grades of excitability, as assigned by ION, differed among DR levels, by using the nonparametric Kruskal-Wallis test. Grades obtained by intraoperative EMG recordings and by physiotherapist observation were studied separately. Moreover, to evaluate whether the excitability grades, as recorded by intraoperative electromyography, were in concordance with the ones evaluated intraoperatively by the physiotherapist, we used Spearman's rank correlation. For the same issue, the kappa coefficient was used to measure the degree of agreement between the electromyogram and physiotherapist's findings.

One-tailed Wilcoxon signed-rank test was used to evaluate whether the absolute difference between the preoperative plan and the final decision for each root level was statistically greater than zero. The criterion $\alpha$ for statistical significance was adjusted using the Bonferroni correction for multiple comparisons to $\alpha=0.01$.

\section{Results}

\section{Demographic Data; Roots Explored and Roots Sectioned}

A total of 13 children with CP participated in the study (age range 6-16 years, average 9 years). The male/female ratio was 11:2 (5.5:1). Seven were diplegic (ambulatory) and 6 were quadriplegic (nonambulatory), as detailed in Table 2.

Data analysis comprised 5 roots (L2-S1) on each side, namely 10 roots for 12 of the 13 patients and 8 roots only for the patient in case 5 (where L-4 was not approached, according to the preoperative chart). When the $\mathrm{S}-2$ roots of the patient in case 3 are added, that is a total of 130 roots.

The percentage of sectioning of the L2-S1 DRs ranged from $33 \%$ to $58 \%$ (mean $48 \%$ ) for the 7 diplegic (ambulatory) children, and from $46 \%$ to $69 \%$ (mean 63\%) for the 6 quadriplegic (nonambulatory) children.

\section{Anatomical and Physiological Information From ION}

Detailed data are the subject of another work, which is currently under preparation. In brief, the successive individual stimulation of VRs and DRs from L-2 to S-2 revealed the innervation territory of the roots (i.e., myotomes) and their overlaps, forming an oblique continuum on the patient's intraoperative sheet as seen in the example reported (Table 1). As expected, the number of muscles that contracted was greater under DR stimulation than after VR stimulation for all radicular levels.

Symmetry of muscle responses, i.e., comparison of myotomes between left and right sides, was also derived from anatomical mapping by VR stimulation. As assessed by electromyography, symmetry was rather poor for roots L-2 and L-3 ( $\mathrm{k}=0.30$ and 0.17 , respectively), but excellent for root L-4 $(\mathrm{k}=0.80)$ and good for roots L-5 and S-1 $(k=$ 0.47 and 0.66 , respectively). In the physiotherapist's evalu- ation there was less variability in the degree of symmetry among the different root levels, with all kappa coefficient values ranging between $0.48-0.70$, i.e., at a fair to good level.

\section{Distribution of Grades of Excitability Among Roots}

Dorsal root excitability correlated with the anatomical level of the stimulated root (Fig. 4). Figure 5 illustrates the difference in excitability grades between different root levels, as evaluated by electromyography (Fig. 5 left) $(\mathrm{p}=$ 0.000036 , Kruskal-Wallis) and by the physiotherapist (Fig. 5 right) ( $\mathrm{p}=0.000012$, Kruskal-Wallis). Root levels corresponding to the upper lumbar segments (L-2 and L-3) were modestly excitable, whereas progressively lower root levels (L-4, L-5, and S-1) displayed higher excitability.

Symmetry of excitability on physiological testing by DR stimulation was good to excellent for all root levels, as assessed by intraoperative electromyography, with a range of kappa coefficient values from 0.56 to 0.89 , with the exception of the L-3 DR, which displayed poor leftright agreement $(\mathrm{k}=0.34)$. The physiotherapist's assessment showed good to excellent symmetry in all root levels, including L-3 DR (kappa coefficient ranging from 0.46 to 0.88).

\section{Global Modifications in Sectioning After ION}

Modifications were performed in 11 of the 13 patients (84\%). Among the 130 roots explored, modifications were carried out in $41.5 \%$ (54 roots). In 27 of the 130 roots, the final percentage of sectioning was higher than the one in the initial evaluation, and in 27 other roots $(20.7 \%)$ it was lower. For all 130 roots, the mean absolute difference in the percentage of sectioning was $10.5 \%$. For the 54 roots in which a modification was decided, the mean absolute difference was $25.3 \%$. For the 27 roots in which a decrease was decided, the mean decrease in sectioning was $26.5 \%$, whereas for the 27 roots in which an increase was decided, the mean increase was $24.1 \%$.

\section{Changes According to Root Levels}

The mean absolute difference in the percentage of sectioning per radicular level was $8.4 \%$ for $\mathrm{L}-2,6.4 \%$ for $\mathrm{L}-3$, $19.6 \%$ for $\mathrm{L}-4,16.5 \%$ for $\mathrm{L}-5$, and $3.2 \%$ for $\mathrm{S}-1$ roots. With regard to radicular level (Fig. 6), decrease in sectioning (bilaterally) from the initial surgical plan involved 8 of the $26 \mathrm{~L}-2$ roots $(30.7 \%), 9$ of the $26 \mathrm{~L}-3$ roots $(34.6 \%), 4$ of the $24 \mathrm{~L}-4$ roots $(16.7 \%), 4$ of the $26 \mathrm{~L}-5$ roots $(15.4 \%)$, and 2 of the $26 \mathrm{~S}-1$ roots $(7.7 \%$ ) (and 0 of the $2 \mathrm{~S}-2$ roots in the patient in case 3 ). Increase in sectioning (bilaterally) was decided in 0 of the $26 \mathrm{~L}-2$ roots $(0 \%), 2$ of the $26 \mathrm{~L}-3$ roots (7.7\%), 11 of the $24 \mathrm{~L}-4$ roots $(45.8 \%), 10$ of the $26 \mathrm{~L}-5$ roots $(38.5 \%), 4$ of the $26 \mathrm{~S}-1$ roots $(15.4 \%)$, and 0 of the 2 S-2 roots. Finally, the initial plan remained unchanged (bilaterally) in 18 of the $26 \mathrm{~L}-2$ roots $(69.2 \%), 15$ of the 26 L-3 roots (57.7\%), 11 of the 24 L-4 roots (45.8\%), 12 of the $26 \mathrm{~L}-5$ roots $(46.1 \%), 20$ of the $26 \mathrm{~S}-1$ roots $(76.9 \%)$, and 0 of the $2 \mathrm{~S}-2$ roots.

Regarding symmetry, the final decision for sectioning was totally symmetrical between the left and right side in 9 patients $(69.2 \%)$. In the other 4 patients $(30.7 \%)$ there was 


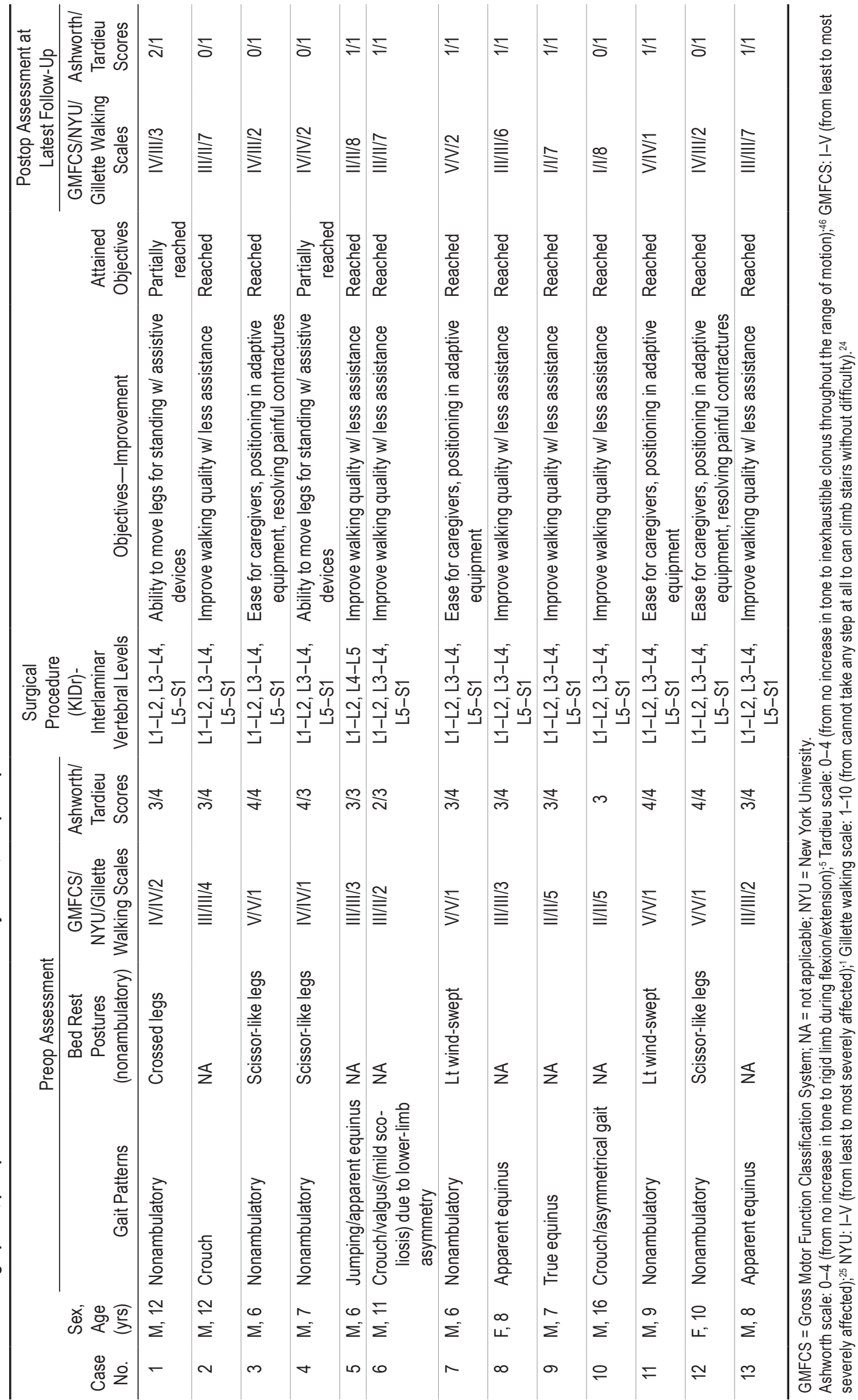




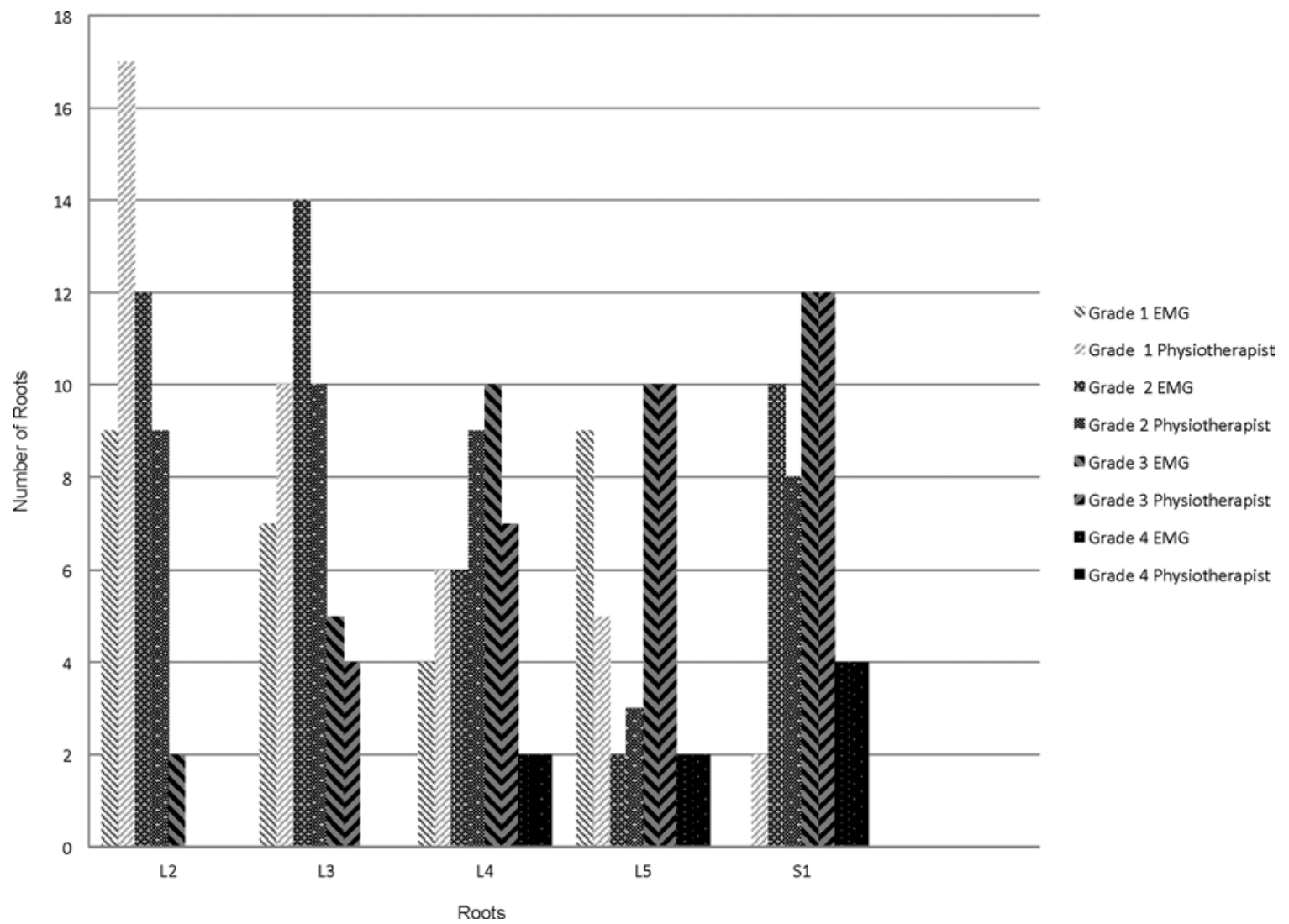

FIG. 4. Bar graph showing the distribution of the motor response of each root, which was recorded and assigned a grade of excitability of 1, 2, 3, or 4 (as described by Fasano and colleagues ${ }^{10-12}$ and modified by Philips and Park ${ }^{35,36}$ and Mittal et al. ${ }^{21}$ ) per radicular level in the 13 patients. Each DR response received a grade from the EMG recordings and the physiotherapist.

asymmetry; it was mild in 3 patients (cases 2,8 , and 10) (23\%) and involved only 1 DR (L-3, L-4, and L-5, respectively), and it was important in 1 patient (case 6) (7.7\%), in whom asymmetry involved 3 DRs (L-3, L-4, and L-5).

Overall, ION resulted in differences in the final percentage of root sectioning for most root levels. For root levels L-2 and L-3 there was a statistically significant absolute difference in the sectioning decision, with mean values of $8.4 \%(p=0.004$, Wilcoxon test) and $6.5 \%(p=$ 0.0004 , Wilcoxon test), respectively. A more noted divergence was observed for roots L-4 and L-5, where the mean value of absolute difference in the sectioning decision was $18 \%(p=0.00003$, Wilcoxon test $)$ and $16.6 \%(p=0.00006$, Wilcoxon test), respectively. Only for the S-1 root was the difference in the sectioning decision slight; it did not reach statistical significance (mean value of absolute difference of 3.1\%; $\mathrm{p}=0.016$, Wilcoxon test with Bonferroni corrected $\alpha=0.01$ ) (Fig. 7).

\section{Comparison Between EMG Recordings and the Physiotherapist's Clinical Observations}

The data of the anatomical mapping after stimulation of each of the 6 VRs showed good agreement between the 2 methods (overall kappa coefficient: 0.64). ${ }^{13}$ Grading of excitability by stimulation of the DR also revealed good to excellent agreement between the intraoperative EMG recordings and the physiotherapist's observations (kappa coefficient: from 0.45 to 0.83$).^{13}$ Appraisals of the excitability grades as observed by the 2 methods were found to be highly similar (Spearman's rank correlation, $\mathrm{p}<0.001$ ) for all roots (Fig. 5). ${ }^{13}$

\section{Discussion \\ Modifications of Final Decision Due to ION}

This prospective study shows that the information from ION led to modifications in the initial surgical plan, which was established by the multidisciplinary team on the basis of the widely accepted concept of sectioning one-half to two-thirds of all DRs from L-2 to S-1. Data provided by ION allowed adjustments in sectioning in as many as 11 of the 13 patients enrolled. In each of these 11 patients, the percentage of sectioning for 4 of the 10 bilaterally targeted roots was modified by approximately $25 \%$. These modifications were not uniform between radicular levels. Decreases in sectioning were most frequently decided for roots L-2 and L-3, and increases for roots L-4 and L-5. The greatest changes involved the L- 4 and L- 5 roots, with increases of $19.6 \%$ and $16.5 \%$, respectively. Data obtained with ION led to increases in the quantity of sectioning indicated in the preoperative chart when muscle responses were grade 4 or 3 and to decreases when monitoring revealed low grades of excitability, i.e., grades 2 or 1. Modifications in sectioning were based on ION revelation of a 

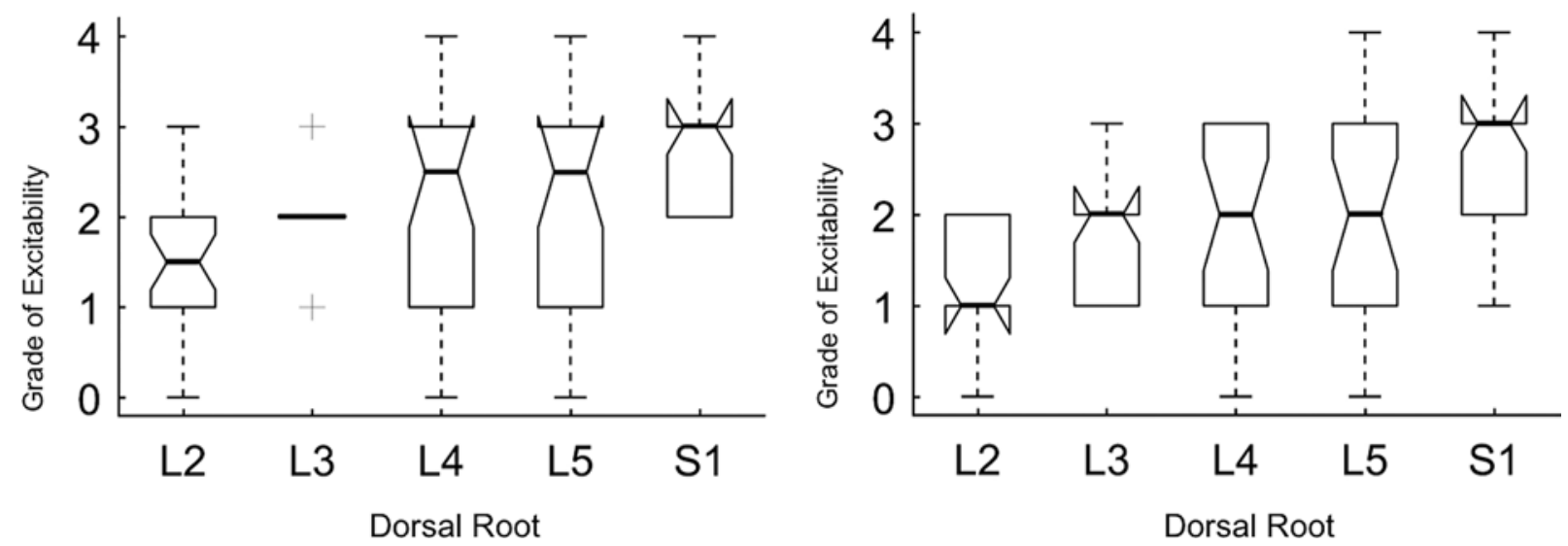

FIG. 5. Box plots showing excitability grade evaluations by intraoperative electromyography (left) and by physiotherapist (right) per DR. The thick line illustrates the median value of excitability grade. The boxes denote the interquartile range. The whiskers denote the minimum and maximum values that fall within 1.5 interquartile ranges below and above the 1 st and 3rd quartile, respectively. Crosses mark the outliers.

significant difference in the degrees of excitability among root levels, with levels L-2 and L-3 displaying lower grades than expected, as opposed to the lower root levels (L-4, L-5, and S-1), which displayed higher excitability.
Classically, since Foerster, ${ }^{14}$ sectioning of root L-4 and to a lesser extent L-5 was traditionally considered functionally risky, due to the antigravity function of L-4 and the innervation of the gluteal muscles by L-5, which were

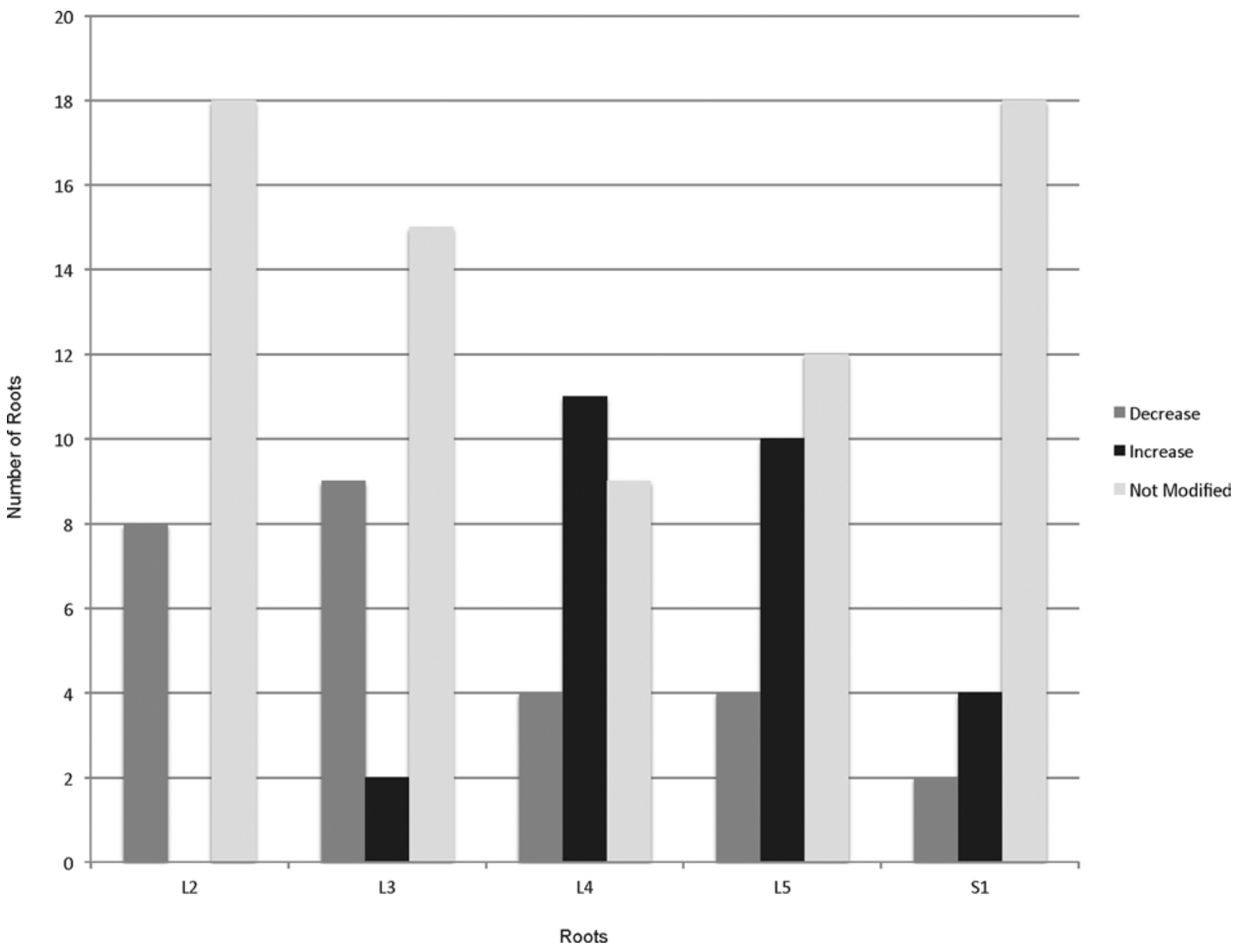

FIG. 6. Bar graph showing the distribution of decrease, increase, and no modification compared to preoperative program in sectioning after ION per radicular level in the 13 patients. 
known to contribute to pelvic stability. This is especially true when those muscles are weak, which is often the case in children with CP. To our knowledge, a systematic comparison of excitability between root levels has not been previously reported per se in the literature.

Another argument in favor of ION, and an incentive to perform it bilaterally, is that a bilateral exploration is more accurate than a unilateral one, which is important in deciding on final sectioning.

\section{Importance of Coexistence of EMG Recordings With Physiotherapist's Observation}

The grading of excitability by EMG recordings was found to be in relatively good correlation with the grading by the physiotherapist, which raises the question of the justification of using both modalities in current practice. On the one hand, the physiotherapist's presence is indispensable during root stimulation to detect responses in unmonitored muscles, among them the iliopsoas muscle, and to identify all responses spreading to distant muscle groups. On the other hand, the EMG recordings offer an objective demonstration of the exact topography of the muscle responses as well as of the degree of hyperactive patterns. Because DRh is a lesioning technique with irreversible results, we consider it safer to benefit from a double control with 2 complementary but not identical tools before deciding on the optimal percentage of root sectioning.

\section{Limitations of the Study}

The main limitation of this study is the relatively small number of patients included. However, because it is a prospective study the exact same protocol was used in all patients. The present study was purposely focused on the specific modifications that ION induced in the initial operative plan; outcome was not considered. However, the overall objectives attained by surgery are mentioned in Table 2.

\section{Controversies Related to Stimulation and Monitoring}

The literature survey reveals a large diversity of opinions about the optimal modality(ies) of ION for children with CP. ${ }^{1-4,6-9,14-17,21-34,37,40-48}$ Several studies even suggest that outcome was not significantly influenced by ION. ${ }^{37,39}$, 44,48 However, most authors agree on the necessity of intraoperative root stimulation to help distinguish DRs from VRs and to define radicular levels and their myotomal innervation.

It is noteworthy that a large number of teams rely on DR stimulation for excitability testing, as recommended by Fasano et al. ${ }^{10-12}$ This has been our strategy also, in addition to checking myotome innervation by anatomical mapping of VRs. For responses that were ambiguous to interpret, the decision was determined on the basis of what was established by the multidisciplinary team and written down in the preoperative chart.

One crucial aspect for studying excitability is the intensity of the current used for stimulation. We have not found any consensus on the parameters for DR stimulation; most teams use rather high intensity - from 6 to $15 \mathrm{~mA}$ (median $10 \mathrm{~mA}$ ). In our investigation we did not exceed $1 \mathrm{~mA}$, to avoid diffusion not only to the neighboring DRs but also to

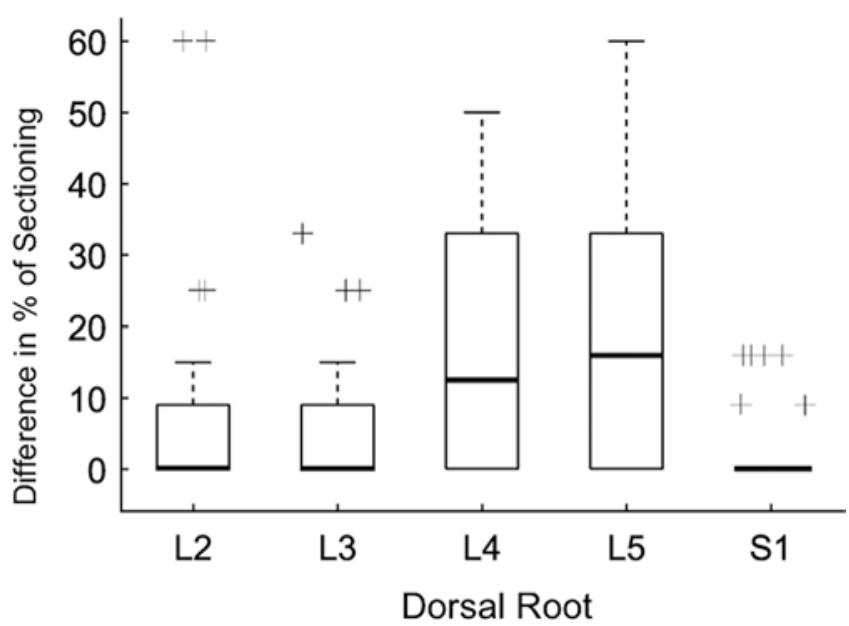

FIG. 7. Box plot of the difference between the preoperative plan and the final decision, in percentage of sectioning at each DR level. The thick line illustrates the median value of difference. The box denotes the interquartile range. The whiskers denote the minimum and maximum values that fall within 1.5 interquartile ranges below and above the 1st and 3rd quartile, respectively. Crosses mark the outliers.

the adjacent VRs. The latter eventuality has been pointed out by Logigian and colleagues, who warned about the fact that high current intensities could produce costimulation of the VR(s) and generate segmental or even extrasegmental pseudoabnormal responses..$^{18-20}$

Another major controversy is whether stimulation and monitoring should be performed at the level of the DR as an entity, or at each of the rootlets of each targeted DR. Theoretically, performing surgery at the individual level of the dorsal rootlets seems in principle more selective than surgery on the DR as a whole.4,6,21,29,30,32-34,47 Fukuhara et al., who conducted a histological study on the sectioned rootlets, claimed that intraoperative monitoring at the rootlet level is a meaningful method because most of the rootlets sectioned showed axonal degeneration on electron microscopy examination. ${ }^{15}$

On the other hand, manipulation of rootlets might modify responses to stimulation, especially when they are individually dissected and held with hooks that inevitably exert some degree of harmful traction. ${ }^{19}$ Because the procedure tends to last longer, it was noted that rootlets become less reactive, to a point at which an inconsistency in responses may occur. ${ }^{36,37,44}$

To our knowledge there exists no systematic anatomical study establishing differences between the various rootlets constituting a DR. Except for the DR entry zone, where there is spatial segregation of sensory axons according to size,$^{42}$ there is no anatomical or physiological evidence to indicate that axons with a particular distribution of central connections are segregated together in the DR more peripherally, which is where the rootlets are generally sectioned. Furthermore, if an abnormal electrophysiological response is observed with tetanic stimulation of a rootlet at threshold, it is not known whether this reflects the functional status of the majority of nerve fibers in that rootlet, or merely the status of the axons with the lowest threshold. ${ }^{45}$

Some experts share the belief that precise identification 
of the roots is not warranted for DRh, because all major lower-extremity muscles in children with spastic CP receive motor innervation from several segments. ${ }^{28}$ From an anatomical point of view, this theory is correct. Nevertheless, each radicular level must be considered as a potential carrier of more or less harmful spasticity.

\section{Conclusions}

Beyond controversies - which all confirm the difficulty of evaluating the importance and reliability of ION-this study reinforces our empirical belief that ION may have a substantial role in answering the fundamental question in DRh surgery: which dorsal roots to section and to what extent. Mapping myotomal innervation by stimulation of both the VRs and DRs, and evaluating the degree of excitability of DRs by stimulation of each root individually, offered a warranty of maximal accuracy. Objective information about the precise degree of excitability of the L-4 and L-5 roots, known to be involved in the antigravity and pelvic stability functions, proved crucial to determining the optimal quantity of sectioning at these levels. This was the most important insight that ION offered in this series of patients.

Obviously ION was not intended to replace but to act synthetically with the preoperative chart that was established within the frame of the pediatric multidisciplinary team. Yet, by modifying significantly the program of the preoperative chart, ION helped to better tailor the surgery to the individual features of each child with CP. Therefore we believe that the methodology used is justified, although time-consuming. Also, a double-check of monitoring by EMG recordings and the physiotherapist's observation, although most frequently concordant, certainly maximizes safety. A larger number of patients will probably help us to progressively optimize this protocol, especially in terms of shortening the duration of the operation.

\section{References}

1. Abbott R: Electrical stimulation in selective dorsal rhizotomy. Adv Neurol 63:263-270, 1993

2. Abbott R: Selective dorsal rhizotomy for the treatment of childhood spasticity, in Sindou M (ed): Practical Handbook of Neurosurgery. Vienna: Springer, 2009, Vol 3, pp 387-395

3. Abbott R: Selective posterior rhizotomy for the treatment of spasticity. Childs Nerv Syst 6:185, 1990

4. Arens LJ, Peacock WJ, Peter J: Selective posterior rhizotomy: a long-term follow-up study. Childs Nerv Syst 5:148-152, 1989

5. Ashworth B: Preliminary trial of carisoprodol in multiple sclerosis. Practitioner 192:540-542, 1964

6. Berman B, Vaughan CL, Peacock WJ: The effect of rhizotomy on movement in patients with cerebral palsy. Am J Occup Ther 44:511-516, 1990

7. Cohen AR, Webster HC: How selective is selective posterior rhizotomy? Surg Neurol 35:267-272, 1991

8. Farmer JP, McNeely PD: Surgery in the dorsal roots for children with cerebral palsy. Oper Tech Neurosurg 7:153-156, 2004

9. Farmer JP, Sabbagh AJ: Selective dorsal rhizotomies in the treatment of spasticity related to cerebral palsy. Childs Nerv Syst 23:991-1002, 2007

10. Fasano VA, Barolat-Romana G, Ivaldi A, Sguazzi A: La radicotomie postérieure fonctionnelle dans le traitement de la spasticité cérébrale Premieres observations sur la stimulation électrique peropératoire des racines postérieures, et leur utilisation dans le choix des racines ã sectionner. Neurochirurgie 22:23-34, 1976

11. Fasano VA, Broggi G, Barolat-Romana G, Sguazzi A: Surgical treatment of spasticity in cerebral palsy. Childs Brain 4:289-305, 1978

12. Fasano VA, Broggi G, Zeme S, Lo Russo G, Sguazzi A: Long-term results of posterior functional rhizotomy. Acta Neurochir Suppl (Wien) 30:435-439, 1980

13. Fleiss JL: Statistical Methods for Rates and Proportions, ed 2. New York: Wiley, 1981

14. Foerster O: On the indications and results of the excision of posterior spinal nerve roots in men. Surg Gynecol Obstet 16:463-474, 1913

15. Fukuhara T, Nakatsu D, Namba Y, Yamadori I: Histological evidence of intraoperative monitoring efficacy in selective dorsal rhizotomy. Childs Nerv Syst 27:1453-1458, 2011

16. Golab MR, Breedon PJ, Vloeberghs M: A wearable headset for monitoring electromyography responses within spinal surgery. Eur Spine J 25:3214-3219, 2016

17. Hays RM, McLaughlin JF, Bjornson KF, Stephens K, Roberts TS, Price R: Electrophysiological monitoring during selective dorsal rhizotomy, and spasticity and GMFM performance. Dev Med Child Neurol 40:233-238, 1998

18. Logigian EL, Shefner JM, Goumnerova L, Scott RM, Soriano SG, Madsen J: The critical importance of stimulus intensity in intraoperative monitoring for partial dorsal rhizotomy. Muscle Nerve 19:415-422, 1996

19. Logigian EL, Soriano SG, Herrmann DN, Madsen JR: Gentle dorsal root retraction and dissection can cause areflexia: implications for intraoperative monitoring during "selective" partial dorsal rhizotomy. Muscle Nerve 24:1352-1358, 2001

20. Logigian EL, Wolinsky JS, Soriano SG, Madsen JR, Scott $\mathrm{RM}$ : H reflex studies in cerebral palsy patients undergoing partial dorsal rhizotomy. Muscle Nerve 17:539-549, 1994

21. Mittal S, Farmer JP, Poulin C, Silver K: Reliability of intraoperative electrophysiological monitoring in selective posterior rhizotomy. J Neurosurg 95:67-75, 2001

22. Newberg NL, Gooch JL, Walker ML: Intraoperative monitoring in selective dorsal rhizotomy. Pediatr Neurosurg 17:124-127, 1991-1992

23. Nordmark E, Josenby AL, Lagergren J, Andersson G, Strömblad LG, Westbom L: Long-term outcomes five years after selective dorsal rhizotomy. BMC Pediatr 8:54, 2008

24. Novacheck TF, Stout JL, Tervo R: Reliability and validity of the Gillette Functional Assessment Questionnaire as an outcome measure in children with walking disabilities. J Pediatr Orthop 20:75-81, 2000

25. Palisano R, Rosenbaum P, Walter S, Russell D, Wood E, Galuppi B: Development and reliability of a system to classify gross motor function in children with cerebral palsy. Dev Med Child Neurol 39:214-223, 1997

26. Park TS, Gaffney PE, Kaufman BA, Molleston MC: Selective lumbosacral dorsal rhizotomy immediately caudal to the conus medullaris for cerebral palsy spasticity. Neurosurgery 33:929-934, 1993

27. Park TS, Johnston JM: Selective dorsal rhizotomy for spastic cerebral palsy, in Goodrich JT (ed): Pediatric Neurosurgery, ed 2. New York: Thieme, 2008, pp 177-183

28. Park TS, Johnston JM: Surgical techniques of selective dorsal rhizotomy for spastic cerebral palsy. Technical note. Neurosurg Focus 21(2):e7, 2006

29. Peacock WJ, Arens LJ: Selective posterior rhizotomy for the relief of spasticity in cerebral palsy. S Afr Med J 62:119124, 1982

30. Peacock WJ, Arens LJ, Berman B: Cerebral palsy spasticity. Selective posterior rhizotomy. Pediatr Neurosci 13:61-66, 1987 
31. Peacock WJ, Nuwer MR, Staudt LA: Dorsal rhizotomy: to monitor or not to monitor? J Neurosurg 80:769-772, 1994

32. Peacock WJ, Staudt LA: Selective posterior rhizotomy: evolution of theory and practice. Pediatr Neurosurg 17:128134, 1991-1992

33. Peacock WJ, Staudt LA: Spasticity in cerebral palsy and the selective posterior rhizotomy procedure. J Child Neurol 5:179-185, 1990

34. Peter JC, Arens LJ: Selective posterior lumbosacral rhizotomy for the management of cerebral palsy spasticity. A 10-year experience. S Afr Med J 83:745-747, 1993

35. Phillips LH, Park TS: Electrophysiologic studies of selective posterior rhizotomy patients, in Park TS, Phillips LH, Peacock WJ (eds): Neurosurgery: State of the Art Reviews. Management of Spasticity in Cerebral Palsy and Spinal Cord Injury. Philadelphia: Hanley \& Belfus, 1989, Vol 4, pp 459-470

36. Phillips LH II, Park TS: Electrophysiologic mapping of the segmental anatomy of the muscles of the lower extremity. Muscle Nerve 14:1213-1218, 1991

37. Pollack MA: Limited benefit of electrophysiological studies during dorsal rhizotomy. Muscle Nerve 17:553-555, 1994

38. Russell DJ, Rosenbaum PL, Avery LM, Lane M: Gross Motor Function Measure (GMFM-66 \& GMFM-88). London: MacKeith, 2002

39. Sacco DJ, Tylkowski CM, Warf BC: Nonselective partial dorsal rhizotomy: a clinical experience with 1-year followUp. Pediatr Neurosurg 32:114-118, 2000

40. Sindou M, Fischer G, Goutelle A, Schott B, Mansuy L: [Selective posterior rhizotomy in the treatment of spasticity.] Rev Neurol (Paris) 130:201-216, 1974 (Fr)

41. Sindou M, Georgoulis G: Keyhole interlaminar dorsal rhizotomy for spastic diplegia in cerebral palsy. Acta Neurochir (Wien) 157:1187-1196, 2015

42. Sindou M, Georgoulis G, Mertens P: Neurosurgical Treatment for Spasticity. A Practical Guide for Children and Adults. Vienna: Springer, 2014

43. Steinbok P: Selective dorsal rhizotomy for spastic cerebral palsy: a review. Childs Nerv Syst 23:981-990, 2007
44. Steinbok P, Kestle JR: Variation between centers in electrophysiologic techniques used in lumbosacral selective dorsal rhizotomy for spastic cerebral palsy. Pediatr Neurosurg 25:233-239, 1996

45. Steinbok P, Tidemann AJ, Miller S, Mortenson P, BowenRoberts T: Electrophysiologically guided versus non-electrophysiologically guided selective dorsal rhizotomy for spastic cerebral palsy: a comparison of outcomes. Childs Nerv Syst 25:1091-1096, 2009

46. Tardieu G: Spasticity: an ill-defined condition. Venice World Fed Neurol 1:24-26, 1983 (Abstract)

47. Turner RP: Neurophysiologic intraoperative monitoring during selective dorsal rhizotomy. J Clin Neurophysiol 26:8284,2009

48. Warf BC, Nelson KR: The electromyographic responses to dorsal rootlet stimulation during partial dorsal rhizotomy are inconsistent. Pediatr Neurosurg 25:13-19, 1996

\section{Disclosures}

The authors report no conflict of interest concerning the materials or methods used in this study or the findings specified in this paper.

\section{Author Contributions}

Conception and design: Georgoulis, Sindou. Acquisition of data: all authors. Analysis and interpretation of data: all authors. Drafting the article: all authors. Critically revising the article: all authors. Reviewed submitted version of manuscript: Georgoulis, Sindou. Approved the final version of the manuscript on behalf of all authors: Georgoulis. Statistical analysis: Georgoulis, Brînzeu. Administrative/technical/material support: all authors. Study supervision: all authors. Surgery: Sindou, Georgoulis.

\section{Correspondence}

George Georgoulis: Agia Sofia Children's Hospital of Athens, Greece.gdgeorgoulis@gmail.com. 